\title{
Dynamics of the Bogie of Maglev Train with Distributed Magnetic Forces
}

\author{
Yaozong Liu, ${ }^{1}$ Wenxi Deng, ${ }^{2}$ and Pu Gong ${ }^{2}$ \\ ${ }^{1}$ College of Mechatronics Engineering and Automation, National University of Defense Technology, Changsha, Hunan 410073, China \\ ${ }^{2}$ Beijing Enterprises Holding Maglev Technology Development Co. Ltd., Changsha Branch, Changsha, Hunan 410073, China \\ Correspondence should be addressed to Yaozong Liu; yzliu@nudt.edu.cn
}

Received 3 June 2015; Revised 3 August 2015; Accepted 6 August 2015

Academic Editor: Jeong-Hoi Koo

Copyright (c) 2015 Yaozong Liu et al. This is an open access article distributed under the Creative Commons Attribution License, which permits unrestricted use, distribution, and reproduction in any medium, provided the original work is properly cited.

\begin{abstract}
A dynamic model of the bogie of maglev train with distributed magnetic forces and four identical levitating controllers is formulated. The vertical, pitching, and rolling degree of freedom of the electromagnet modules and their coupling are considered. The frequency responses of the bogie to track irregularity are investigated with numerical simulation. The results tell us that there are resonances related to the first electromagnetic suspension whose frequencies are determined by the control parameters. A comparative analysis has been carried out between the models with distributed or concentrated magnetic forces. The comparison indicates that simplifying the distributed magnetic force to concentrated one degenerates the dynamic behavior of the maglev bogie, especially resulting in overestimated resonances of the first electromagnetic suspension of maglev trains. The results also indicate that those resonances only occur on specific wavelengths of irregularity that relate to the length of the electromagnets.
\end{abstract}

\section{Introduction}

Due to its lower noise, less costly maintenance, and no danger of derailment, the maglev train is currently under rapid development around the world. A commercial line of high speed maglev trains has been operated in Shanghai, China, since 2003. Commercial lines of low speed maglev trains have been built in both Japan and Korea. Two new commercial lines of low speed maglev transportation are under construction in China. The maglev train is levitated by the magnetic forces between the electromagnets and the track which are adjusted by controllers in real time for stable levitation. Hence, the magnetic forces are the most fundamental elements while modeling the dynamics of maglev trains. The magnetic forces are obvious distributed along the electromagnets. But in the most of the literatures, they are simplified to concentrated forces for convenience of modeling. Liu et al. [1] proposed a proportional-differential (PD) controller with fractional orders to enhance the levitation stiffness around the operating point. The single degree of freedom (SDOF) maglev model with linearized magnetic force (LMF) was adopted. Zhou et al. [2] employed an improved least mean square algorithm with phase correction to suppress the self-excited vibration of the maglev train due to the flexibility of the track, who simplified the maglev model in the same way. Kong et al. [3] formulated sliding mode controllers (SMCs) for the whole vehicle model with three identical SDOF bogie models with LMFs to enhance the dynamic response of the maglev system for various speeds. Li et al. [4] derived feedback linearization controllers with acceleration feedback and disturbance observer for the maglev train with five bogies to improve robustness, who modeled the magnetic forces along one module of the bogie as two concentrated nonlinear forces. He et al. [5] designed a decoupling controller for the maglev module which was modeled as a rigid beam with two concentrated forces applied at fixed positions. Even in the more detailed virtual prototype simulation model of maglev train, the levitation forces are modeled as concentrated ones [6]. In earlier articles, the actively controlled electromagnetic forces are substituted with a sequence of equivalent springs and dashpots to represent the distribution $[7,8]$. Owing to the simplicity, such dynamic models are significantly helpful for us to understand the dynamical behavior of maglev trains and to design and compare different control laws. But the 


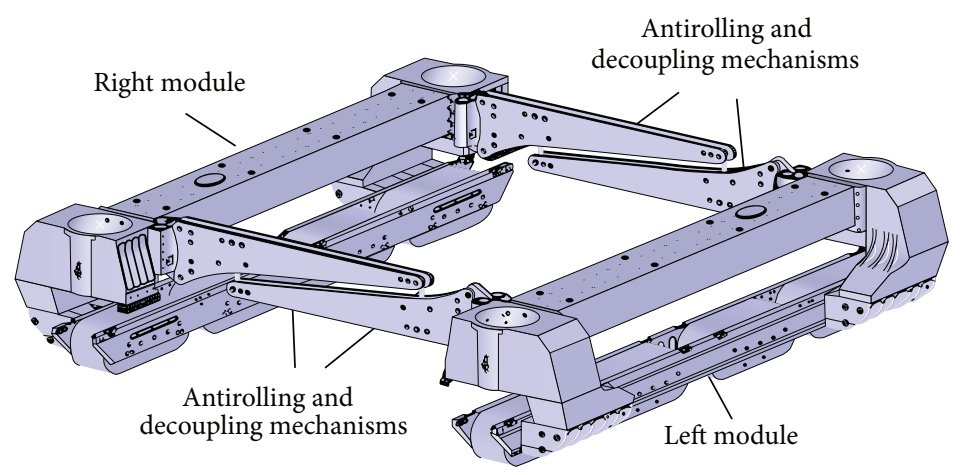

FIGURE 1: The three-dimensional structure of the maglev bogie.

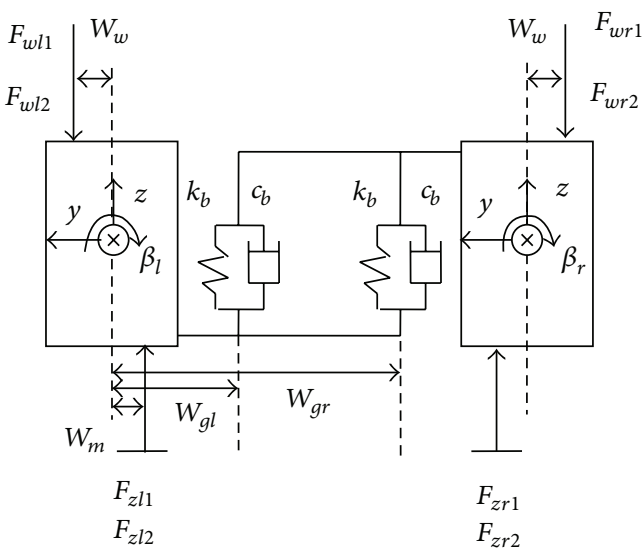

(a) Forces of the maglev bogie from rear view

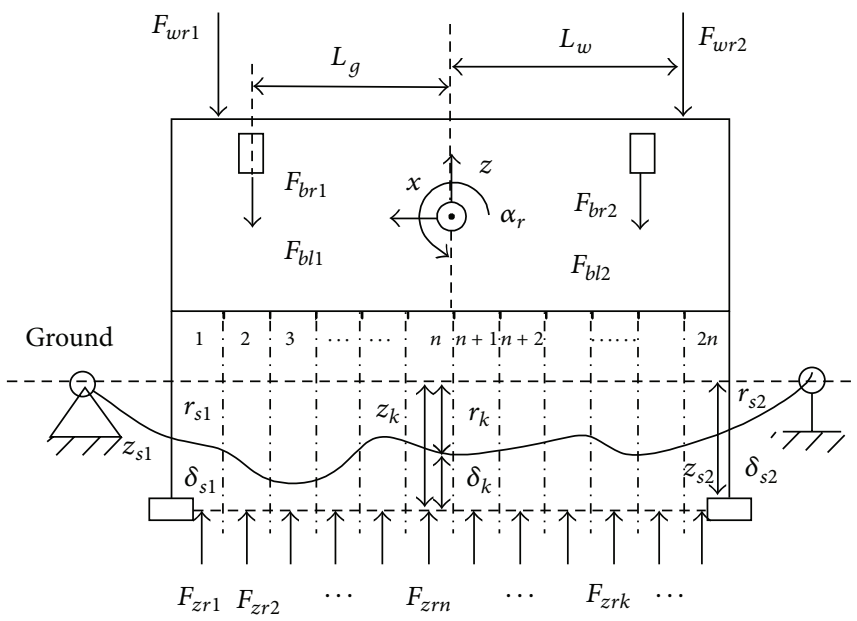

(b) Forces of the right module from side view

FIGURE 2: Sketch of the maglev bogie on track.

simplified dynamic model raises the robustness requirements of the controller and is not suitable for investigating the dynamic responses of the vehicle.

In the full scale low speed maglev trains, the electromagnets are arranged in modules under the train along the track. There are four or five bogies under each vehicle. Each bogie has one electromagnet module in each side. The module is meters long and consists of several coils with common pole plates. The coils in one module are divided into two sets for the connivance of controlling, which results in the fact that each individually controlled electromagnetic force is distributed more than one meter along the pole plate. Since it is inversely proportional to the square of the levitation gap, the distributed magnetic force will produce very different moments to the module in contrast to the fabricated concentrated one, especially while the module pitched or yawed from the track.

In this paper, a dynamic model of the bogie of maglev train with distributed magnetic forces is formulated. The frequency responses of the bogie to track irregularity in different traveling speed are presented, analyzed, and compared to those from the model with concentrated magnetic forces.

\section{Modeling}

The maglev bogie is an independent unit to levitate and propel the cabin of maglev train. It consists of two rigid modules mounted with electromagnets and linear inductive motors and two antirolling and decoupling mechanisms (see Figure 1). The authors formulated the dynamic model of the maglev bogie with concentrated magnetic forces in [9]. The magnetic force of each controlled point is simplified as a fabricated concentrated one acting at the center of electromagnets. In this paper, we reformulated the dynamic model of the maglev bogie with distributed magnetic forces. The following assumptions are made while we formulate the dynamic equations:

(1) The distribution of the magnetic fields is uniform along the whole pole face of the electromagnets. The magnetic leakage and edge effect of the electromagnet are neglected.

(2) The electromagnets module is a rigid body so that the levitation gaps of its subsections can be determined by the gaps of the module ends and the known track irregularity. 


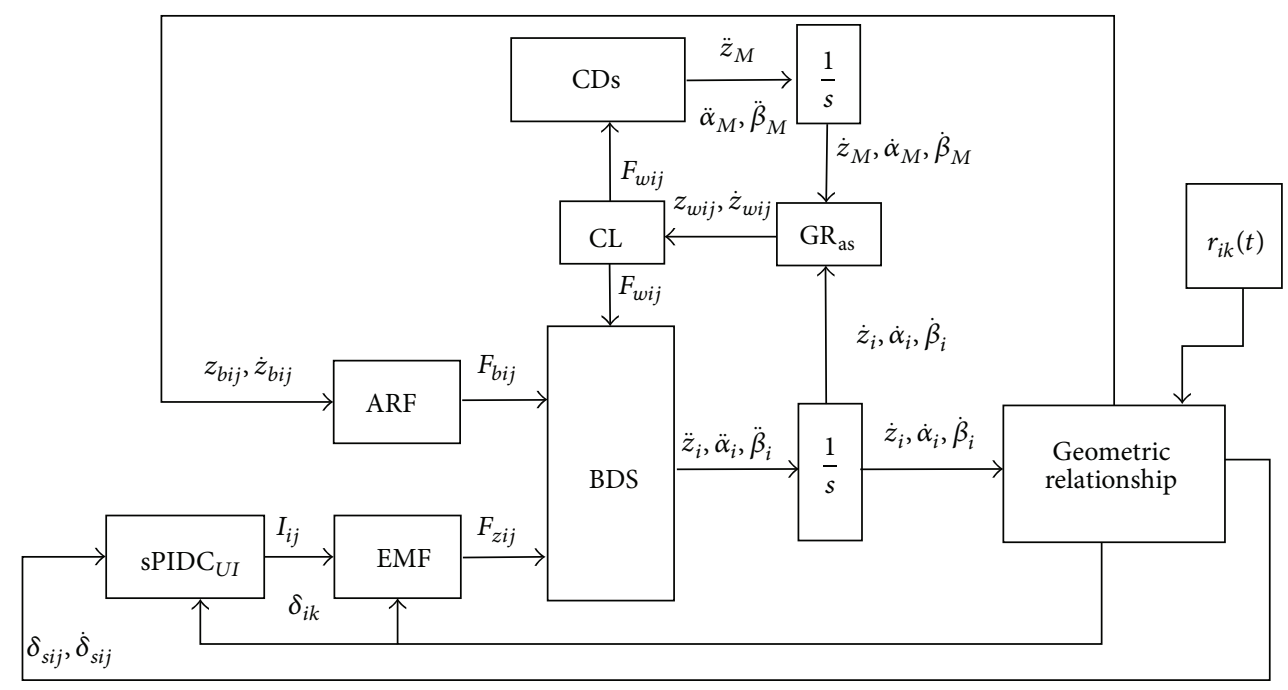

FIGURE 3: Block diagram of the maglev bogie model.
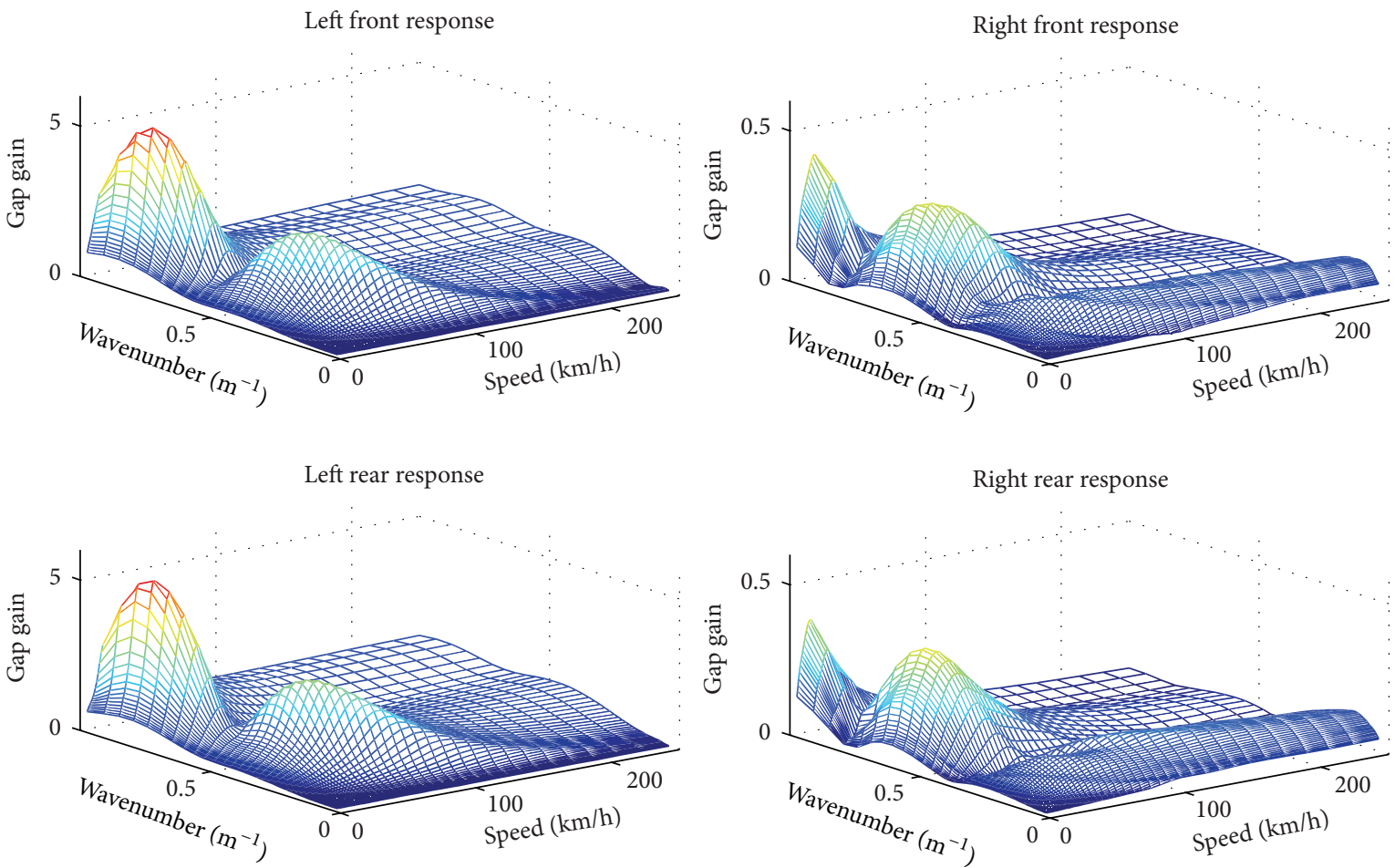

FIGURE 4: Levitation gap gains of the maglev bogie modeled with concentrated magnetic forces traveling through tracks with different wavenumbers' irregularity in different speeds. The bogies are controlled with four identical decentralized PID controllers with parameters of $K_{p}=4973, K_{i}=64959, K_{d}=84$, and $K_{c}=932$.

(3) The track is considered to be stiff, so the flexible distortion of the track can be neglected.

2.1. Kinematics and Dynamics of the Maglev Bogie. The forces diagrams of the maglev bogie are present in Figure 2. $F_{w l 1}$, $F_{w l 2}, F_{w r 1}$, and $F_{w r 2}$ are cabin loads acted on the left front, left rear, right front, and right rear levitation point. $F_{w l k}$ and $F_{w r k}(k=1,2, \ldots, 2 n)$ are the magnetic forces of each subsection of the electromagnets. $W_{w}$ is the distance in $y$ direction from the mass center of module to the load force. $W_{m}$ is the distance in $y$ direction from the mass center of each module to its magnetic force. $k_{b}$ is the stiffness and $c_{b}$ is the damp of the antirolling bar in the antirolling and decoupling mechanism. $W_{g l}$ and $W_{g r}$ are the distances in $y$ direction from the mass center of module to the adjacent and nonadjacent antirolling bar. $L_{g}$ and $L_{w}$ are the distance in $x$ direction from the mass center of module to the antirolling force and to the cabin loads, respectively. $F_{b l 1}, F_{b r 1}, F_{b l 2}$, and $F_{b r 2}$ are the 
Left front response

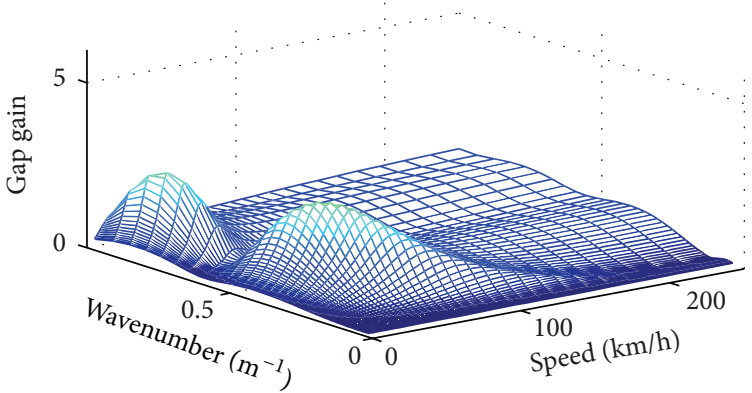

Left rear response

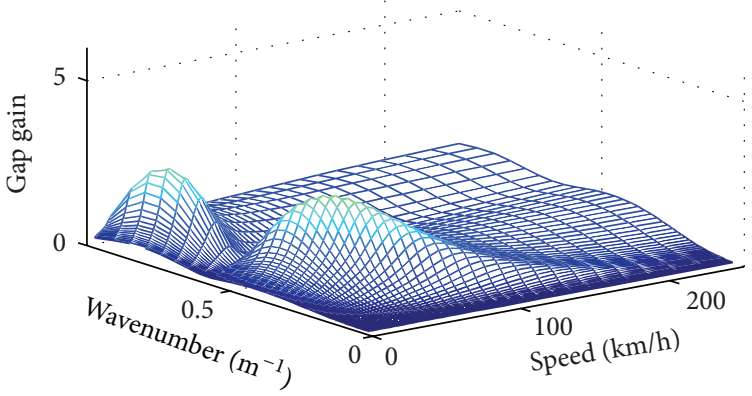

Right front response

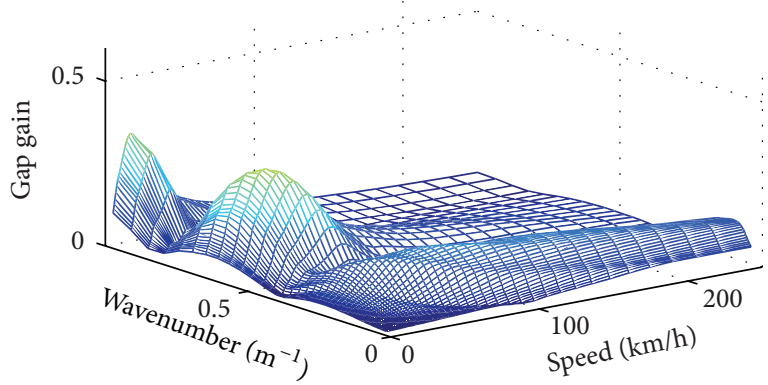

Right rear response

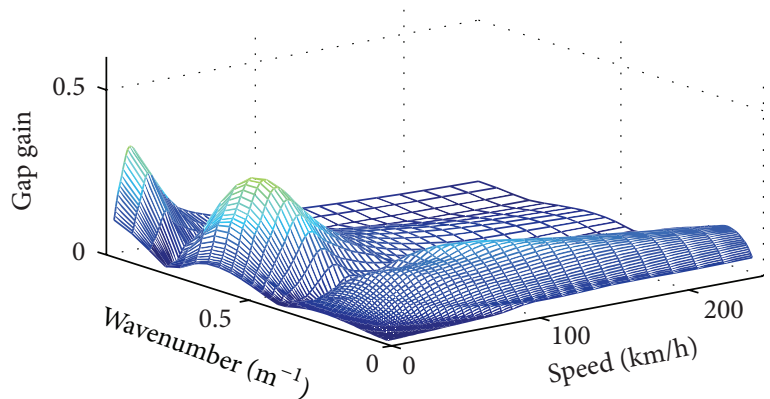

FIGURE 5: Levitation gap gains of the maglev bogie modeled with distributed magnetic forces traveling through tracks with different wavenumbers' irregularity in different speeds. The bogies are controlled with four identical decentralized PID controllers with parameters of $K_{p}=4973, K_{i}=64959, K_{d}=84$, and $K_{c}=932$.

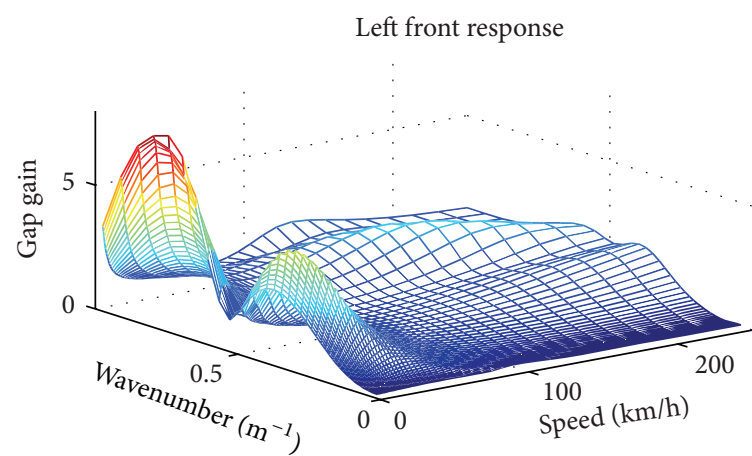

Left rear response

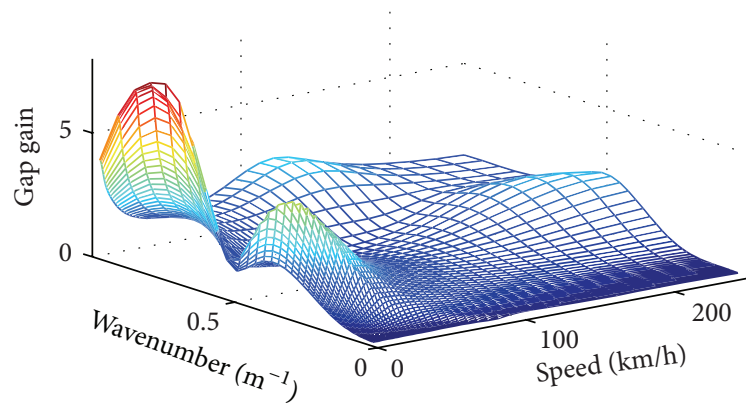

Right front response

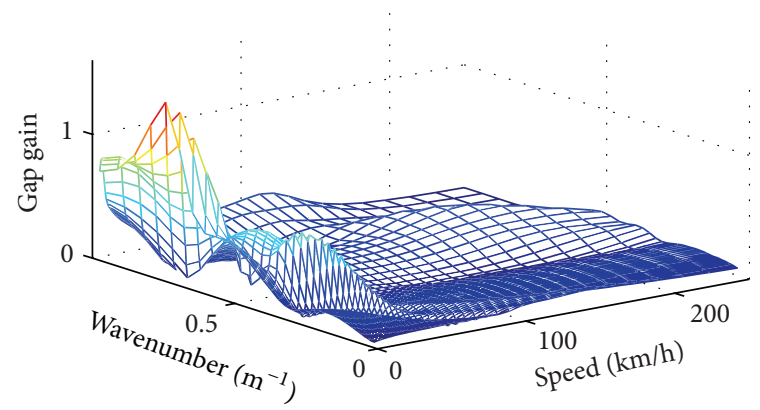

Right rear response

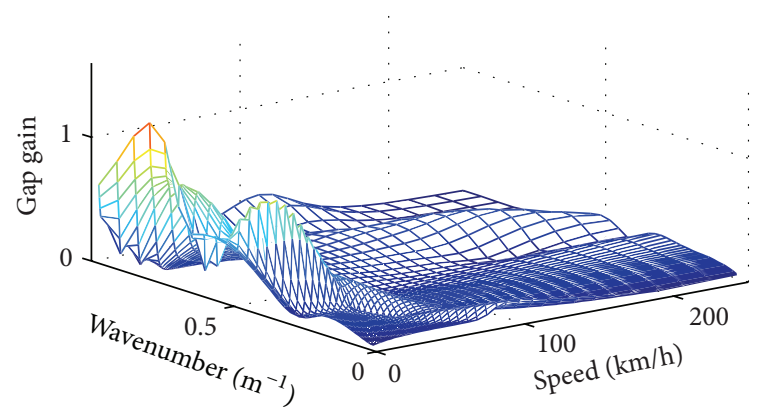

FIGURE 6: Levitation gap gains of the maglev bogie modeled with concentrated magnetic forces traveling through tracks with different wavenumbers' irregularity in different speeds. The bogies are controlled with four identical decentralized PID controllers with parameters of $K_{p}=4400, K_{i}=5000, K_{d}=100$, and $K_{c}=40$. 


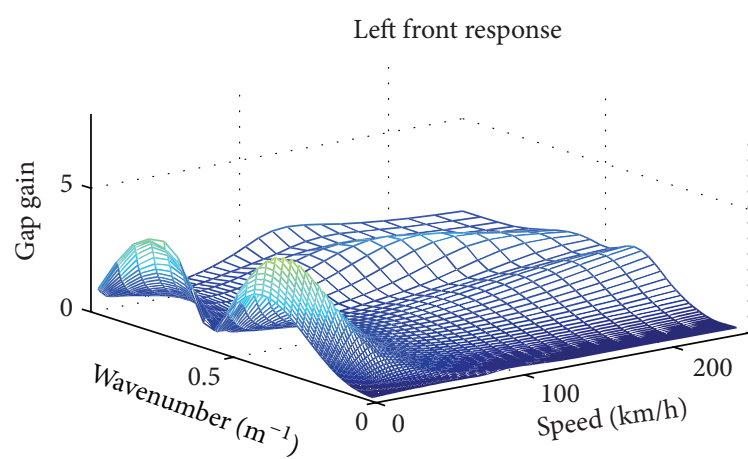

Left rear response

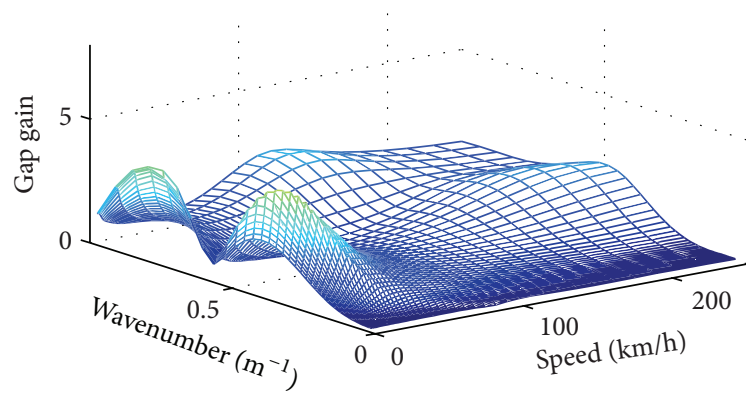

Right front response

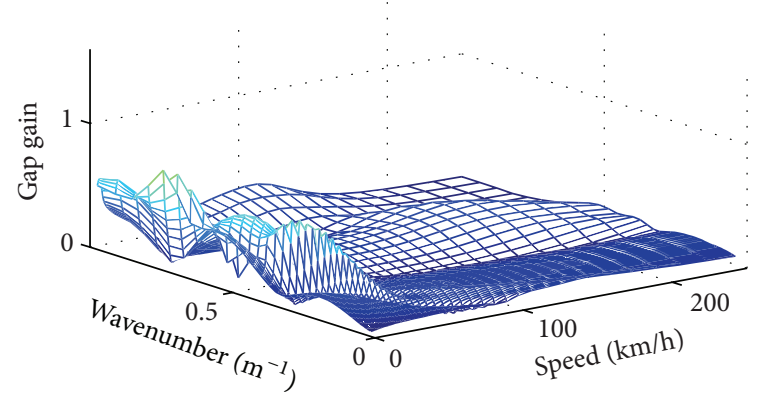

Right rear response

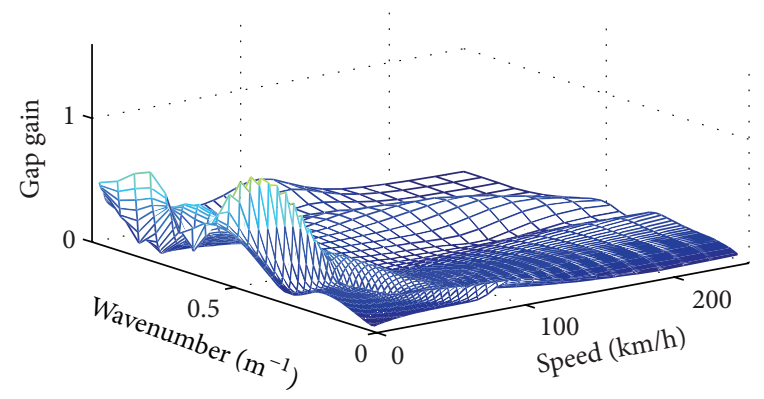

FIGURE 7: Levitation gap gains of the maglev bogie modeled with distributed magnetic forces traveling through tracks with different wavenumbers' irregularity in different speeds. The bogies are controlled with four identical decentralized PID controllers with parameters of $K_{p}=4400, K_{i}=5000, K_{d}=100$, and $K_{c}=40$.

antirolling forces produced by the antirolling and decoupling mechanisms linking the left and right module. Figure 2(b) presents the forces of the right module from side view. The forces of the left module can be obtained in the same way.

When the current through the coil of the electromagnet is $I$ and the levitation gap between the electromagnet and the lower surface of the steel track is $\delta$, the electromagnetic force $F_{z}$ is given by

$$
F_{z}=\frac{\mu_{0} N^{2} A}{4} \frac{I^{2}}{\delta^{2}}
$$

where $N$ is the number of turns of a single electromagnet, $A$ is the area of the magnetic pole, and $\mu_{0}$ is the space permeability. Because the levitation gap is not the same at different position along the electromagnet and the magnetic forces are distributed along it, we need to split the electromagnet into $2 n$ segments and consider that each electromagnetic force $F_{z i k}$ can be described by (1). Therefore, the distributed magnetic forces can be expressed as follows:

$$
\begin{aligned}
& F_{z i k}=\frac{\mu_{0} N^{2} A_{k}}{4} \frac{I_{i j}^{2}}{\delta_{i k}^{2}}, \\
& \delta_{i k}=r_{i k}-z_{i k}, \\
& z_{i k}= \frac{(4 n-2 k+1) z_{s i 1}+(2 k-1) z_{s i 2}}{4 n}, \\
& i=l, r j= \begin{cases}1, & \text { when } k=1,2, \ldots, n, \\
2, & \text { when } k=n+1, \ldots, 2 n .\end{cases}
\end{aligned}
$$

The subscript $i=l, r$ indicates the left or right electromagnets module. The subscript $j=1,2$ indicates the front or rear of the module. The subscript $k=1,2, \ldots, 2 n$ indicates the subsection of the electromagnet. $A_{k}$ is the area of the magnetic pole of $k$ th subsection of electromagnet. $r_{i k}$ is the irregularity error of the steel track's surface above $k$ th subsection of electromagnet. $z_{i k}$ is the vertical displacement of $k$ th subsection of electromagnet. Given $n=1$ so that $k=1,2$, the distributed magnetic forces are degenerate to be concentrated ones. $z_{s i 1}, z_{s i 2}$ are the front or rear end heave of the electromagnets module which can be determined by geometric relationship of its vertical displacement, pitch angle, and roll angle:

$$
\begin{aligned}
& z_{s l 1}=z_{l}-\frac{\alpha_{l} L_{x}}{2}+\beta_{l} W_{m}, \\
& z_{s l 2}=z_{l}+\frac{\alpha_{l} L_{x}}{2}+\beta_{l} W_{m}, \\
& z_{s r 1}=z_{r}-\frac{\alpha_{r} L_{x}}{2}-\beta_{r} W_{m}, \\
& z_{s r 2}=z_{r}+\frac{\alpha_{r} L_{x}}{2}-\beta_{r} W_{m}, \\
& \dot{z}_{s i j}=\frac{d z_{s i j}}{d t},
\end{aligned}
$$

$$
i=l, r j=1,2 \text {, }
$$



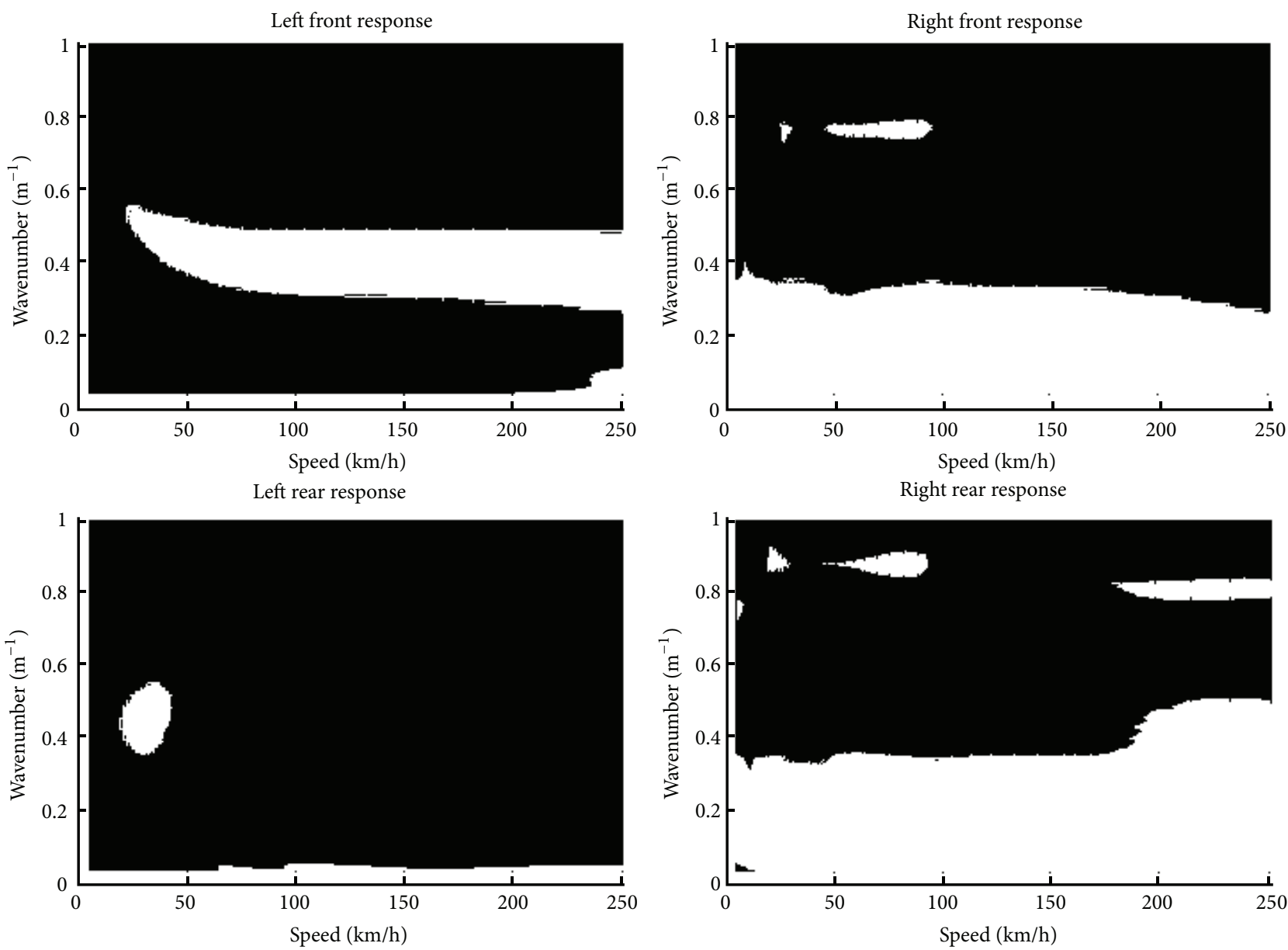

FIGURE 8: Indication with black filling of the levitation gap gains from the model with concentrated magnetic forces larger than those from the distributed magnetic forces. The filling ratios in the figure are $82 \%$ for the left front point, $69 \%$ for the right front point, $96 \%$ for the left rear point, and $60 \%$ for the right rear point. The bogies are controlled with four identical decentralized PID controllers with parameters of $K_{p}=4973, K_{i}=64959, K_{d}=84$, and $K_{c}=932$.

where $z_{i}, \alpha_{i}$, and $\beta_{i}$ are the vertical displacement, the pitch angle, and the roll angle at the mass center of the electromagnets modules. $L_{x}$ is the length of the electromagnet.

The antirolling forces $F_{b i j}$ can be expressed as follows:

$$
F_{b i j}=k_{b} z_{b i j}+c_{b} \dot{z}_{b i j}, \quad i=l, r j=1,2
$$

where $z_{b i j}$ is the deformation of the antirolling bars, which can be determined by geometric relationships in (5) of the vertical displacement, the pitch angle, and the roll angle of the modules:

$$
\begin{aligned}
& z_{b r 1}=z_{r}-\alpha_{r} L_{g}+\beta_{r} W_{g l}-z_{l}+\alpha_{l} L_{g}+\beta_{l} W_{g r}, \\
& z_{b l 1}=z_{r}-\alpha_{r} L_{g}+\beta_{r} W_{g r}-z_{l}+\alpha_{l} L_{g}+\beta_{l} W_{g l}, \\
& z_{b r 2}=z_{r}+\alpha_{r} L_{g}+\beta_{r} W_{g l}-z_{l}-\alpha_{l} L_{g}+\beta_{l} W_{g r},
\end{aligned}
$$

$$
\begin{aligned}
& z_{b l 2}=z_{r}+\alpha_{r} L_{g}+\beta_{r} W_{g r}-z_{l}-\alpha_{l} L_{g}+\beta_{l} W_{g l}, \\
& \dot{z}_{b i j}=\frac{d z_{b i j}}{d t}, \begin{array}{l} 
\\
\\
\end{array} \quad i=l, r \quad j=1,2 .
\end{aligned}
$$

The cabin loads $F_{w i j}$ can be expressed as follows:

$$
F_{w i j}=k_{a} z_{w i j}+c_{a} \dot{z}_{w i j}, \quad i=l, r j=1,2,
$$

where $z_{w i j}$ is the deformation of the airspring, which can be determined by geometric relationships in (7) of the vertical displacement, the pitch angle, and the roll angle of the modules and the cabin:

$$
\begin{aligned}
z_{w l 1}= & z_{l}-\frac{\alpha_{l} L_{w}}{2}+\beta_{l} W_{w} \\
& -\left(z_{M}-\frac{\alpha_{M} L_{w}}{2}+\beta_{M} W_{w M}\right),
\end{aligned}
$$



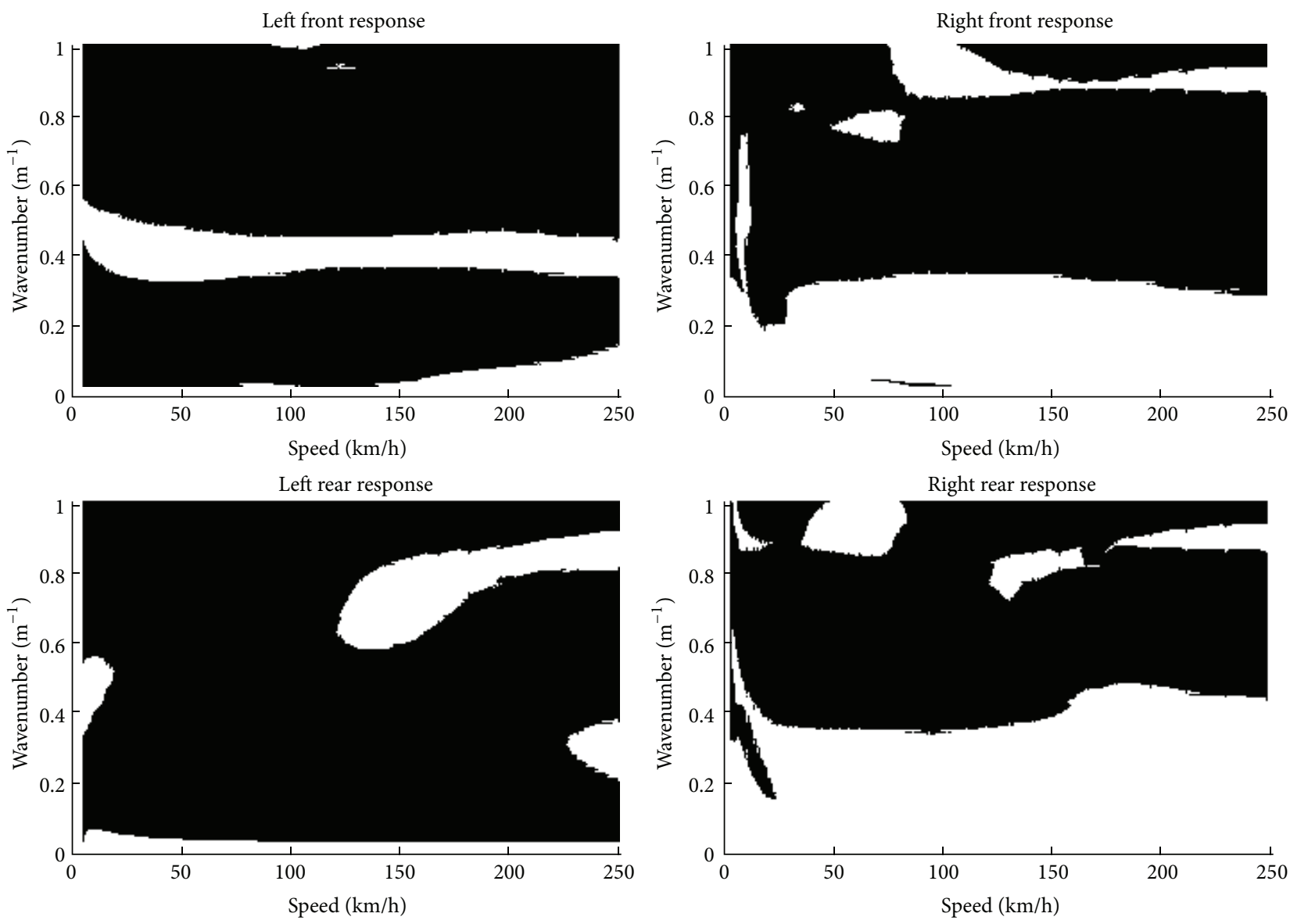

FIGURE 9: Indication with black filling of the levitation gap gains from the model with concentrated magnetic forces larger than those from the distributed magnetic forces. The filling ratios are $84 \%$ for the left front point, $62 \%$ for the right front point, $89 \%$ for the left rear point, and $55 \%$ for the right rear point. The bogies are controlled with four identical decentralized PID controllers with parameters of $K_{p}=4400$, $K_{i}=5000, K_{d}=100$, and $K_{c}=40$.

$$
\begin{aligned}
z_{w l 2}= & z_{l}+\frac{\alpha_{l} L_{w}}{2}+\beta_{l} W_{w} \\
& -\left(z_{M}+\frac{\alpha_{M} L_{w}}{2}+\beta_{M} W_{w M}\right), \\
z_{w r 1}= & z_{r}-\frac{\alpha_{r} L_{w}}{2}-\beta_{r} W_{w} \\
& -\left(z_{M}-\frac{\alpha_{M} L_{w}}{2}-\beta_{M} W_{w M}\right), \\
z_{w r 2}= & z_{r}+\frac{\alpha_{r} L_{w}}{2}-\beta_{r} W_{w} \\
& -\left(z_{M}+\frac{\alpha_{M} L_{w}}{2}-\beta_{M} W_{w M}\right), \\
\dot{z}_{w i j}= & \frac{d z_{w i j}}{d t} .
\end{aligned}
$$

center of the cabin, which can be determined by the dynamic equations of the cabin as follows:

$$
\begin{aligned}
& M \cdot \ddot{z}_{M}=-M g+F_{w l 1}+F_{w l 2}+F_{w r 1}+F_{w r 2}, \\
& J_{\alpha M} \ddot{\alpha}_{M}=\left(-F_{w l 1}+F_{w l 2}-F_{w r 1}+F_{w r 2}\right) L_{w M}, \\
& J_{\beta M} \ddot{\beta}_{M}=\left(-F_{w l 1}-F_{w l 2}+F_{w r 1}+F_{w r 2}\right) W_{w M},
\end{aligned}
$$

where $J_{\alpha M}$ and $J_{\beta M}$ are the inertia of the pitch movement and the roll movement of the cabin. $W_{w M}$ is the distance in $y$ direction from the mass center of cabin to the center of airspring. $L_{w M}$ is the distance in $x$ direction from the mass center of cabin to the center of airspring.

For the levitation dynamics of the maglev bogie, we consider the vertical, pitch, and roll movements. The dynamic equations of the maglev bogie are as follows:

$W_{w M}$ is the distance in $y$ direction from the mass center of the cabin to the load force. $z_{M}, \alpha_{M}$, and $\beta_{M}$ are the vertical displacement, the pitch angle, and the roll angle at the mass

$$
\begin{aligned}
m_{l} \ddot{z}_{l}= & -m_{l} g-F_{w l 1}-F_{w l 2}+F_{b l 1}+F_{b l 2}+F_{b r 1}+F_{b r 2} \\
& +\sum_{k=1}^{2 n} F_{z l k}
\end{aligned}
$$




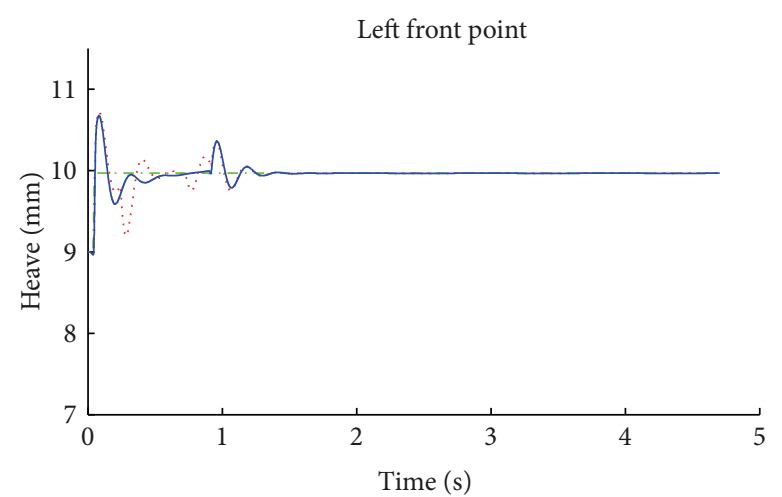

... Concentrated force

...- Reference

— Distributed force

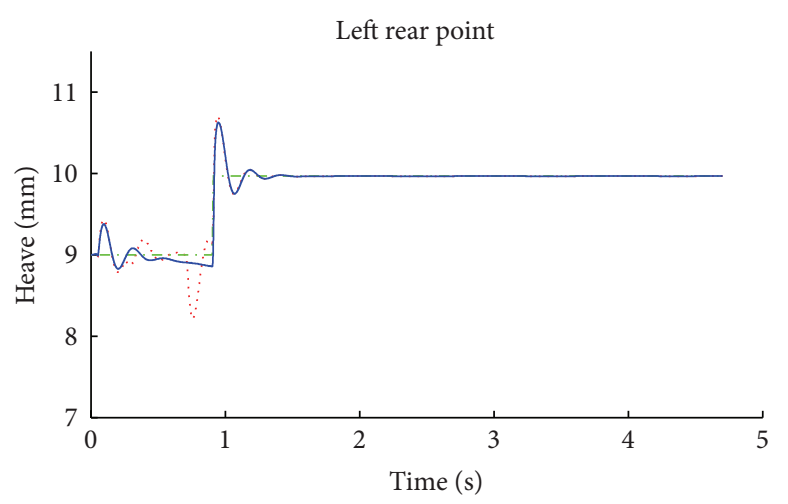

Concentrated force

...- Reference

— Distributed force

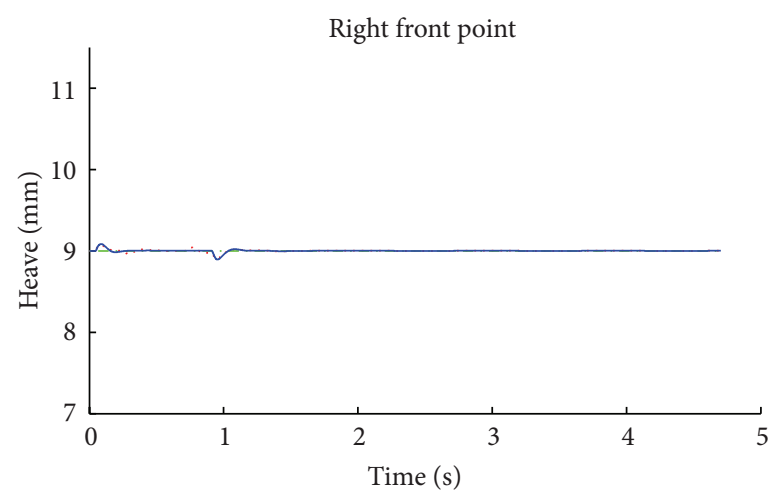

... Concentrated force

-.. Reference

— Distributed force

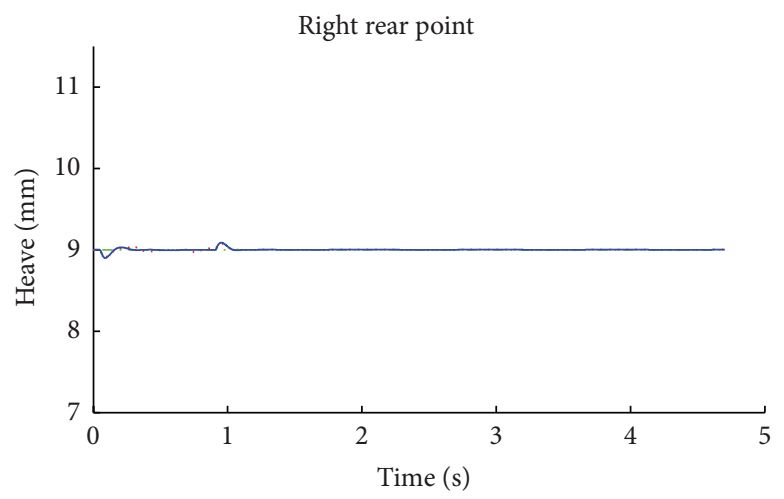

Concentrated force

-. - Reference

— Distributed force

FIGURE 10: Step responses of the maglev bogie with concentrated or distributed magnetic forces. The largest overshot occurs at left front point. The overshot from the concentrated force model is $1.35 \mathrm{~mm}$ against $1 \mathrm{~mm}$ step uprise of the track while the overshot from the distributed force model is $0.93 \mathrm{~mm}$.

$$
\begin{aligned}
m_{r} \ddot{z}_{r}= & -m_{r} g-F_{w r 1}-F_{w r 2}-F_{b l 1}-F_{b l 2}-F_{b r 1} \\
& -F_{b r 2}+\sum_{k=1}^{2 n} F_{z r k}, \\
J_{\alpha l} \ddot{\alpha}_{l}= & \left(F_{w l 1}-F_{w l 2}\right) L_{w} \\
& -\left(F_{b l 1}+F_{b r 1}-F_{b l 2}-F_{b r 2}\right) L_{g} \\
& -\sum_{k=1}^{2 n} F_{z l k}\left(\frac{2 n+1-2 k}{4 n} L_{x}\right), \\
J_{\alpha r} \ddot{\alpha}_{r}= & \left(F_{w r 1}-F_{w r 2}\right) L_{w} \\
& +\left(F_{b l 1}+F_{b r 1}-F_{b l 2}-F_{b r 2}\right) L_{g} \\
& -\sum_{k=1}^{2 n} F_{z r k}\left(\frac{2 n+1-2 k}{4 n} L_{x}\right), \\
J_{\beta l} \ddot{\beta}_{l}= & -\left(F_{w l 1}+F_{w l 2}\right) W_{w}-\left(F_{b l 1}+F_{b l 2}\right) W_{g l}
\end{aligned}
$$

$$
\begin{aligned}
& -\left(F_{b r 1}+F_{b r 2}\right) W_{g r}-\sum_{k=1}^{2 n} F_{z l k} W_{m}, \\
J_{\beta r} \ddot{\beta}_{r}= & +\left(F_{w r 1}+F_{w r 2}\right) W_{w}-\left(F_{b l 1}+F_{b l 2}\right) W_{g l} \\
& -\left(F_{b r 1}+F_{b r 2}\right) W_{g r}+\sum_{k=1}^{2 n} F_{z r k} W_{m},
\end{aligned}
$$

where $J_{\alpha i}, J_{\beta_{i}}$ are the inertia of the pitch movement and the roll movement of the module.

2.2. Magnetic Levitation Model of Single Point. The maglev bogie is controlled with four identical decentralized controllers. For a single electromagnet levitation point, we usually adopt PID controller to regulate the desired current:

$$
\begin{aligned}
I_{e i j}=I_{0}+K_{p}\left(\delta_{s i j}-\delta_{0}\right)+K_{d} \dot{\delta}_{s i j}+K_{i} \int & \delta_{s i j}, \\
& i=l, r j=1,2 .
\end{aligned}
$$



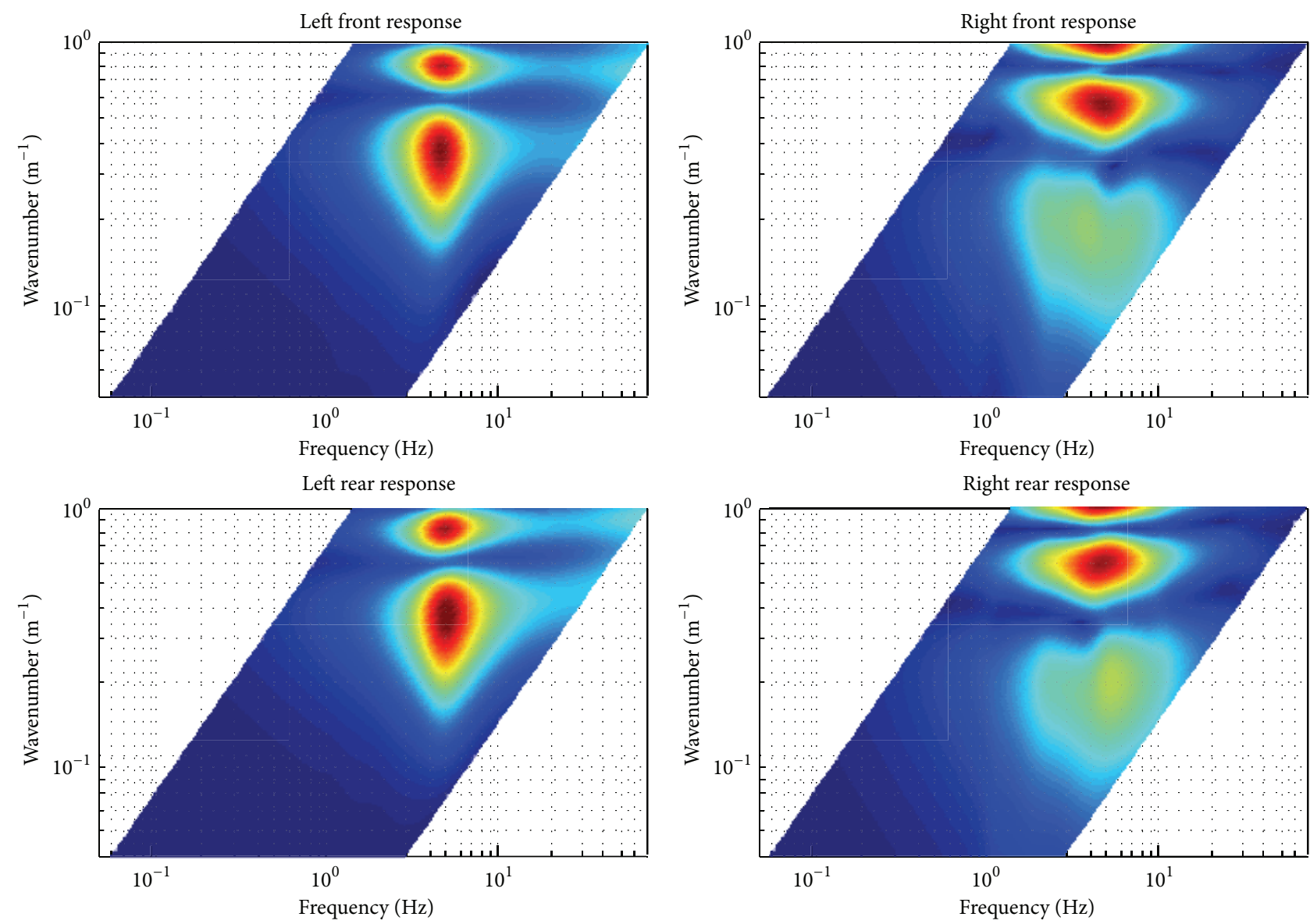

FIGURE 11: Meshes from top view of the levitation gap gains of the maglev bogie on the frequency and wavenumber axes in log scale. This figure corresponds to Figure 5.

If the levitation system can be stabilized at the desired levitation gap $\delta_{0}$ and the steady-state current is $I_{0}$, we call $\left(\delta_{0}, I_{0}\right)$ the equilibrium point or operating point.

Since the time delay of the current is unnegligible due to the inductance of the coil, another proportional controller for current loop is adopted to generate the desired current:

$$
U_{i j}=R I_{0}+K_{c}\left(I_{e i j}-I_{i j}\right), \quad i=l, r j=1,2,
$$

where $R$ is the resistance of the coil and $K_{c}$ is the proportional coefficient of the current loop.

In addition, the relationship between the control voltage $U$ and the current $I$ can be described as

$$
\begin{aligned}
& U_{i j}(t)=R I_{i j}(t)+N \frac{d \phi_{i j}(t)}{d t}, \\
& \phi_{i 1}(t)=\sum_{k=1}^{n} \frac{\mu_{0} N A I_{i 1}(t)}{2 \delta_{i k}(t)}, \\
& \phi_{i 2}(t)=\sum_{k=n+1}^{2 n} \frac{\mu_{0} N A I_{i 2}(t)}{2 \delta_{i k}(t)},
\end{aligned}
$$

2.3. Magnetic Levitation Model of the Bogie. For each levitation point in the maglev bogie, we adopt the same PID controller with proportional current feedback. According to the expressions above, the block diagram of the maglev bogie model with controllers is shown in Figure 3, in which EMF represents electromagnetic forces equations (2), ARF represents antirolling forces equations (4), CL represents cabin loads equations (6), CDs represents cabin dynamics equations (8), BDs represents bogie dynamics equations (9) (11), sPIDC $_{U I}$ represents four identical single point controllers by (12) (14), $\mathrm{GR}_{\mathrm{as}}$ represents the geometric relationship of the airsping's deformations by (7), and the geometric relationship block translates the motion of electromagnets modules to the levitation gaps and relative velocities of the subsections of electromagnet by (3) as well as to the deformations of antirolling bars by (5).

\section{Simulation and Discussion}

Adding ideal sine irregularities with different wavenumber (spatial frequency) to the left track, the dynamical responses of the bogie traveling in different speeds are simulated. The amplitude of the sine irregularity is specified as $0.1 \mathrm{~mm}$. The wavenumber varies from 0.02 to $1 \mathrm{~m}^{-1}$, which corresponds to 

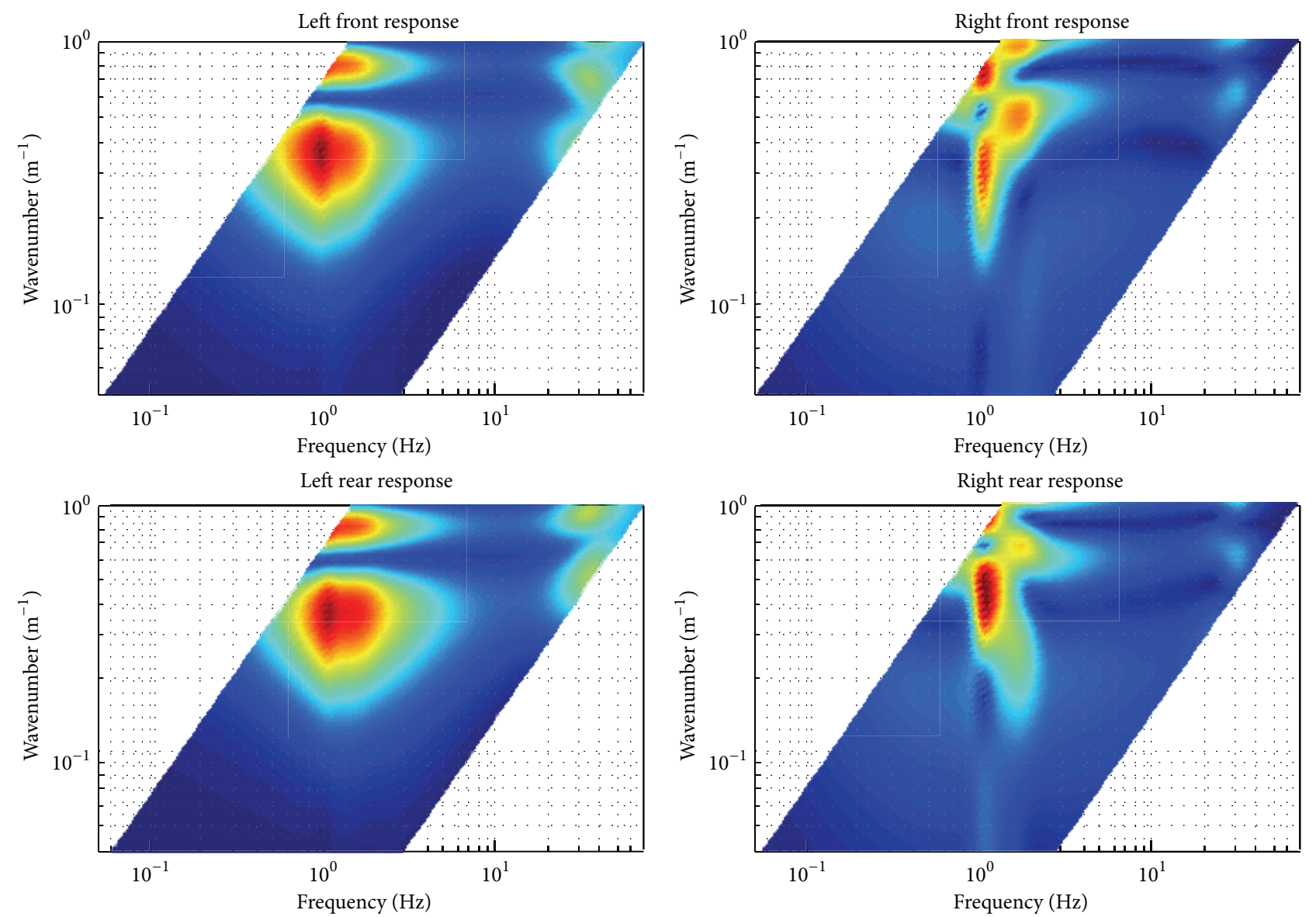

FIGURE 12: Meshes from top view of the levitation gap gains of the maglev bogie on the frequency and wavenumber axes in log scale. This figure corresponds to Figure 7.

the irregularity with wavelength (spatial period) from $50 \mathrm{~m}$ to $1 \mathrm{~m}$. The parameters of the bogie and cabin used in simulation are shown in Table 1. The dynamic responses of the levitation modules to such ideal sine irregularity are gap variations in sine wave. The amplitude ratio of the gap variation and the track irregularity is calculated, which represents the frequency response function of the bogie to different track irregularity in different speed.

It should be pointed out that the more subsections the electromagnets were divided, the more noticeable the differences between the frequency responses from both models were presented, and the more the simulation time was needed. After repeated attempts, we found that 20 subsections are enough to give converged results. It took about $73 \%$ more computation time than the concentrated force model to finish the simulation presented in the following sections, which is affordable.

3.1. The Coupling Effects of Modules in the Maglev Bogie. Figures 4 to 7 show the levitation gap gains of the four decentralized controlled points on the bogie with concentrated or distributed magnetic forces and different control parameters. It can be seen from those figures that the gap gains of the front point and the rear point in same module are quite alike on both modules while the dynamic response of different module is quite different. Because the irregularity is applied only to the left track, the gap gains of the left module are much larger than those of the right module, which indicates that the antirolling and decoupling mechanisms in the bogie do decouple both modules significantly. Since there is no irregularity in the right track, the dynamic responses of the right module are coupled from the left module through the antirolling and decoupling mechanisms, which mean that those mechanisms do slightly couple both modules. The shapes of the gap gain meshes of the right module are quite different from those of the left module. In lower speed range there are three peaks in the gap gain meshes of the right module in contrast to the two peaks of the left module. In higher speed range, the gap gains of the right module for long wavelength irregularity (small wavenumbers) are larger than those for short wavelength, which are smaller on the left module. The differences indicate that the coupling effects of the antirolling and decoupling mechanisms are related to frequencies of the dynamics of modules. More details can be found in [9]. From the frequency response meshes of the right module, we can find the secondary suspension resonance at very low frequency, especially obvious in Figures 6 and 7. But it is almost unobservable in the meshes of the left module 
TABLE 1: Parameter values used in simulation.

\begin{tabular}{|c|c|c|}
\hline Parameter & Description & Value \\
\hline$m_{l}, m_{r}$ & Mass of maglev module & $800 \mathrm{~kg}$ \\
\hline$J_{\alpha l}, J_{\alpha r}$ & Pitch inertia of maglev module & $477 \mathrm{kgm}^{2}$ \\
\hline$J_{\beta l}, J_{\beta r}$ & Rolling inertia of maglev module & $88 \mathrm{kgm}^{2}$ \\
\hline$W_{m}$ & Lateral distance from the mass center of maglev module to magnetic force & $43 \mathrm{~mm}$ \\
\hline$W_{w}$ & Lateral distance from the mass center of maglev module to cabin load & $53 \mathrm{~mm}$ \\
\hline$W_{g l}$ & Lateral distance from the mass center of maglev module to the adjacent antirolling bar & $553 \mathrm{~mm}$ \\
\hline$W_{g r}$ & Lateral distance from the mass center of maglev module to the nonadjacent antirolling bar & $1363 \mathrm{~mm}$ \\
\hline$L_{g}$ & Longitudinal distance from the mass center of maglev module to the antirolling bar & $964 \mathrm{~mm}$ \\
\hline$L_{w}$ & Longitudinal distance from the mass center of maglev module to the cabin load & $1125 \mathrm{~mm}$ \\
\hline$L_{x}$ & Length of the electromagnet of levitation point & $1325 \mathrm{~mm}$ \\
\hline$k_{b}$ & Stiffness of the antirolling bar & $300 \mathrm{~N} / \mathrm{mm}$ \\
\hline$c_{b}$ & Damping of the antirolling bar & $1000 \mathrm{Ns} / \mathrm{m}$ \\
\hline$M$ & Mass of the cabin & $5400 \mathrm{~kg}$ \\
\hline$J_{\alpha M}$ & Pitch inertia of cabin & $3200 \mathrm{kgm}^{2}$ \\
\hline$J_{\beta M}$ & Rolling inertia of cabin & $2826 \mathrm{kgm}^{2}$ \\
\hline$W_{w M}$ & Lateral distance from the mass center of cabin to the center of airspring & $1010 \mathrm{~mm}$ \\
\hline$L_{w M}$ & Longitudinal distance from the mass center of cabin to the center of airspring & $1125 \mathrm{~mm}$ \\
\hline$k_{a}$ & Stiffness of airspring & $60 \mathrm{~N} / \mathrm{mm}$ \\
\hline$c_{b}$ & Damping of airspring & $2000 \mathrm{Ns} / \mathrm{m}$ \\
\hline$N$ & Number of turns of the electromagnet coil & 360 turns \\
\hline$A$ & Area of the magnetic pole & $0.0183 \mathrm{~m}^{2}$ \\
\hline$\mu_{0}$ & Space permeability & $4 \pi \cdot 10^{-7} \mathrm{H} / \mathrm{m}^{2}$ \\
\hline$R$ & Resistance of the coil & $0.56 \mathrm{ohm}$ \\
\hline
\end{tabular}

because the gap gain of the irregularity resonance is much larger than the secondary suspension resonance.

\subsection{The Influences of the Magnetic Force Distribution on the} Dynamics of the Maglev Bogie. In order to investigate the effects of the force distribution on the dynamics of the bogie, let us compare Figure 4 to Figure 5 and Figure 6 to Figure 7. It can be found out that the shapes of the meshes from the distributed forces model are almost the same as their counterparts from the concentrated forces model. The most noticeable difference is that the amplitudes of those peaks in lower speed range and short wavelength irregularities are obviously smaller when the magnetic forces were modeled as distributed forces. The distribution nature of the magnetic forces flattened the levitation gap gain meshes. In fact most of the gap gains are decreased. For convenience of comparison, we stack the gap gain meshes from both models with the same control parameters in one figure and set to be top view. The mesh faces from the model with concentrated forces are set to be black and those from the distributed forces model are set to be white. The results are present in Figures 8 and 9 . The black regions identify where the gap gains are decreased in the distributed forces model while the white regions mean that they are increased. The black filling ratios in Figure 8 are 82\% for the left front point, $69 \%$ for the right front point, $96 \%$ for the left rear point, and $60 \%$ for the right rear point, respectively. The black filling ratios are $84 \%$ for the left front point, $62 \%$ for the right front point, $89 \%$ for the left rear point, and 55\% for the right rear point in Figure 9. Such results mean that the simplified concentrated magnetic forces degenerate the dynamic behavior of the maglev bogie, especially resulting in overestimated resonances of the first electromagnetic suspension of maglev trains. In other words, the vibration of the modules and the cabin will be overestimated if the simulation model employed the concentrated magnetic forces instead of distributed forces.

Figure 10 shows the step responses from both models. The bogie passes a $1 \mathrm{~mm}$ uprise on the left track at $t=0 \mathrm{~s}$ at the speed of $10 \mathrm{~km} / \mathrm{h}$. The heave displacements of the modules are investigated as the responses. It is clearly present that the step responses from the concentrated forces model have larger overshot and more staggering transience than those from the distributed forces model. The overshot of the model with concentrated forces is $135 \%$, while the overshot of the distributed force model is $93 \%$. It also can be seen that the disturbance does not transfer to the right module very much, which is consistent with the much smaller gap gains of the right module.

3.3. The Characteristics of the Dynamics of the Maglev Bogie. There are noticeable peaks much larger than 1 in the gap gain meshes of Figures 5 and 7, which mean that the bogie resonates to the specified track irregularity in special speed ranges. Since the gap gains are mainly determined by the stiffness and damping of the first electromagnetic suspension, the control parameters have significant influence on the 
gap gains, which can be seen by comparing Figure 5 to Figure 7 . The shapes of the gap gain meshes are quite different especially in high speed range.

In order to understand the dynamics essence of the gap gain meshes of the maglev bogie, we translate the coordinate of traveling speed to frequency and redraw the gap gain meshes from top view so that different amplitudes of the gap gain are present in different colors. Figures 11 and 12 correspond to Figures 5 and 7, respectively. It can be quickly recognized that those peaks in the meshes are aligned on the same frequencies which are determined by the control parameters. The frequency is the natural frequency of the first electromagnetic suspension. In our simulation frequency range up to $70 \mathrm{~Hz}$, the first group parameters $K_{p}=4973$, $K_{i}=64959, K_{d}=84$, and $K_{c}=932$ result in a natural frequency at about $4.5 \mathrm{~Hz}$ and the second group parameters $K_{p}=4400, K_{i}=5000, K_{d}=100$, and $K_{c}=40$ determined the natural frequency of about $1.3 \mathrm{~Hz}$. It also should be noticed that the large gap gains form several resonance islands instead of a line aligning on the natural frequency in these figures. It means that the resonance relates to the wavelength of irregularity as well as the natural frequency. Further investigation tells us that the resonances only occur on the intersection points of the natural frequency and the irregularities whose wavelength is once or twice the length of the electromagnets. Since the gap sensor is mounted at the end of the electromagnet and detected the variation of the track there, the controller changed the current to keep the levitation gap while in fact it was not necessary because the average gap of the whole electromagnets did not change very much as well as the distributed magnetic forces. Although the natural frequencies are different due to the different control parameters in Figures 11 and 12, the gap gain peaks are on the same wavenumbers of the irregularity. As to the maglev track, the most significant periodic irregularity is the sleeper spacing of the track [10]. According to the above conclusion, the sleeper spacing of the maglev track should be carefully kept away from the length of the electromagnets.

\section{Conclusion}

In this paper, the dynamic equations of the bogie of maglev train with distributed magnetic forces and four identical levitating controllers are formulated. The vertical, pitching, and rolling degree of freedom of the maglev modules in the bogie and their coupling are taken into account. The frequency responses of the bogie to track irregularity are investigated with numerical simulation. The comparative analysis between the models with distributed or concentrated magnetic forces indicates that simplifying the distributed magnetic force to concentrated one degenerates the dynamic behavior of the maglev bogie, especially resulting in overestimated resonances of the first electromagnetic suspension of maglev trains. The gap gains of the bogie tell us that there are resonances related to the first electromagnetic suspension especially in low speed range. The resonance frequencies are determined by the control parameters. The most interesting phenomena are those resonances that only occur on specific wavelength of irregularity that relate to the length of the electromagnets.

\section{Conflict of Interests}

The authors declare that there is no conflict of interests regarding the publication of this paper.

\section{Acknowledgments}

This work is supported by the National Key Technology R\&D Program of the 12th Five-Year Plan and study on application and integration demonstration of the medium-low speed maglev transportation, 2012BAG07B01.

\section{References}

[1] H. Liu, X. Zhang, and W. Chang, "PID control to maglev train system," in Proceedings of the International Conference on Industrial and Information Systems (IIS '09), pp. 341-343, April 2009.

[2] D. F. Zhou, C. H. Hansen, and J. Li, "Application of least mean square algorithm to suppression of maglev track-induced selfexcited vibration," Journal of Sound and Vibration, vol. 330, no. 24, pp. 5791-5811, 2011.

[3] E. Kong, J.-S. Song, B.-B. Kang, and S. Na, "Dynamic response and robust control of coupled maglev vehicle and guideway system," Journal of Sound and Vibration, vol. 330, no. 25, pp. 6237-6253, 2011

[4] J.-H. Li, J. Li, and G. Zhang, "A practical robust nonlinear controller for maglev levitation system," Journal of Central South University, vol. 20, no. 11, pp. 2991-3001, 2013.

[5] G. He, J. Li, and P. Cui, "Decoupling control design for the module suspension control system in maglev train," Mathematical Problems in Engineering, vol. 2015, Article ID 865650, 13 pages, 2015.

[6] K. J. Kim, J. B. Han, H. S. Han, and S. J. Yang, "Coupled vibration analysis of maglev vehicle-guideway while standing still or moving at low speeds," Vehicle System Dynamics, vol. 53, no. 4, pp. 587-601, 2015.

[7] C. F. Zhao and W. M. Zhai, "Maglev vehicle/guideway vertical random response and ride quality," Vehicle System Dynamics, vol. 38, no. 3, pp. 185-210, 2002.

[8] H. G. Dai, Dynamic behavior of maglev vehicle/guideway system with control [Ph.D. dissertation], Case Western Reserve University, 2005.

[9] Y.-Z. Liu, W.-X. Deng, and P. Gong, "Dynamics-control modeling and analysis for bogie of low-speed maglev train," Journal of the China Railway Society, vol. 36, no. 9, pp. 39-43, 2014 (Chinese).

[10] G. Zhang, J. Li, and Z.-J. Yang, "Estimation of power spectrum density track irregularities of low-speed maglev railway lines," Journal of the China Railway Society, vol. 33, no. 10, pp. 73-78, 2011 (Chinese). 

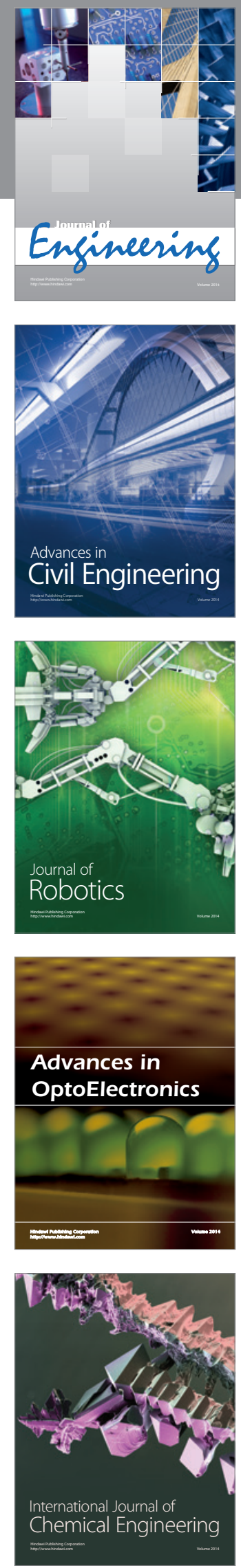

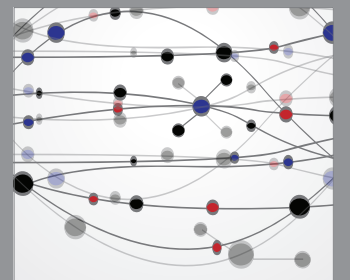

The Scientific World Journal
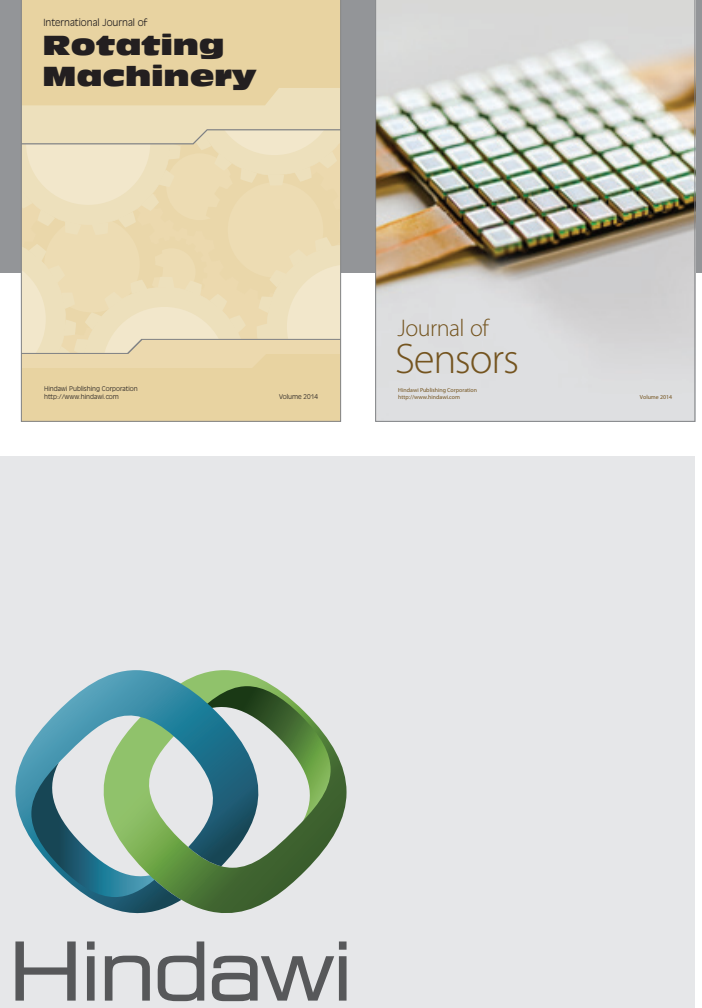

Submit your manuscripts at http://www.hindawi.com
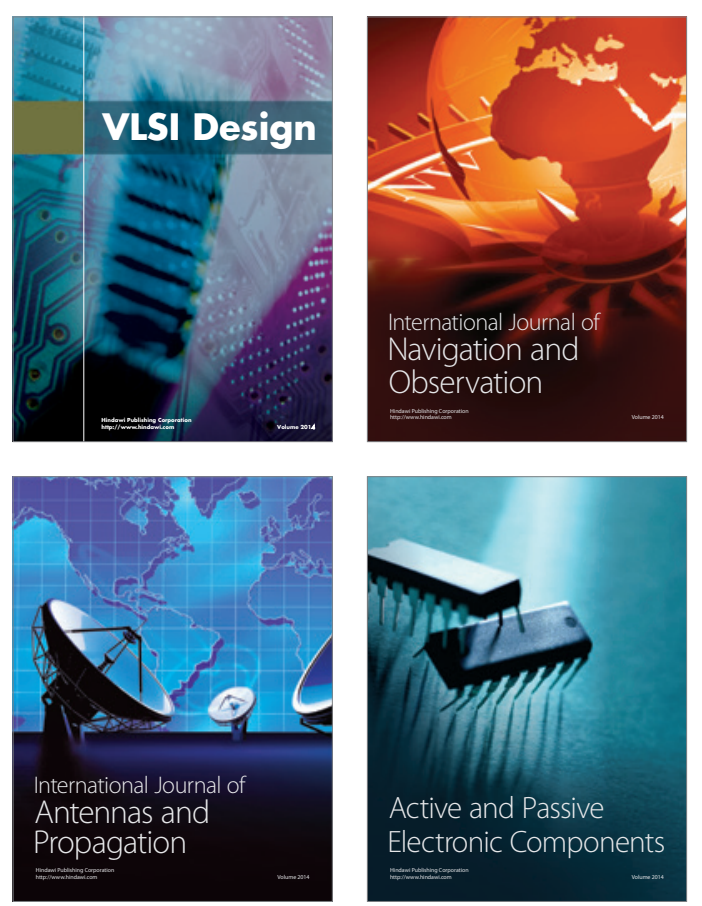
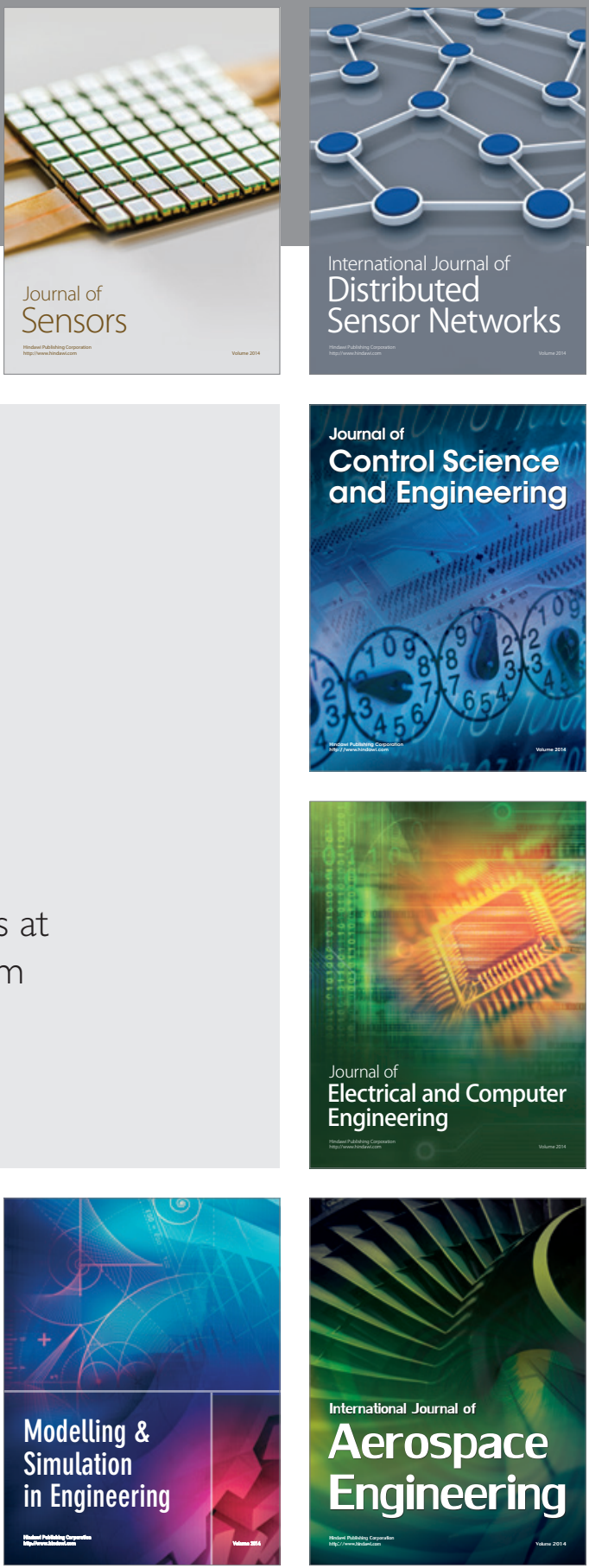

Journal of

Control Science

and Engineering
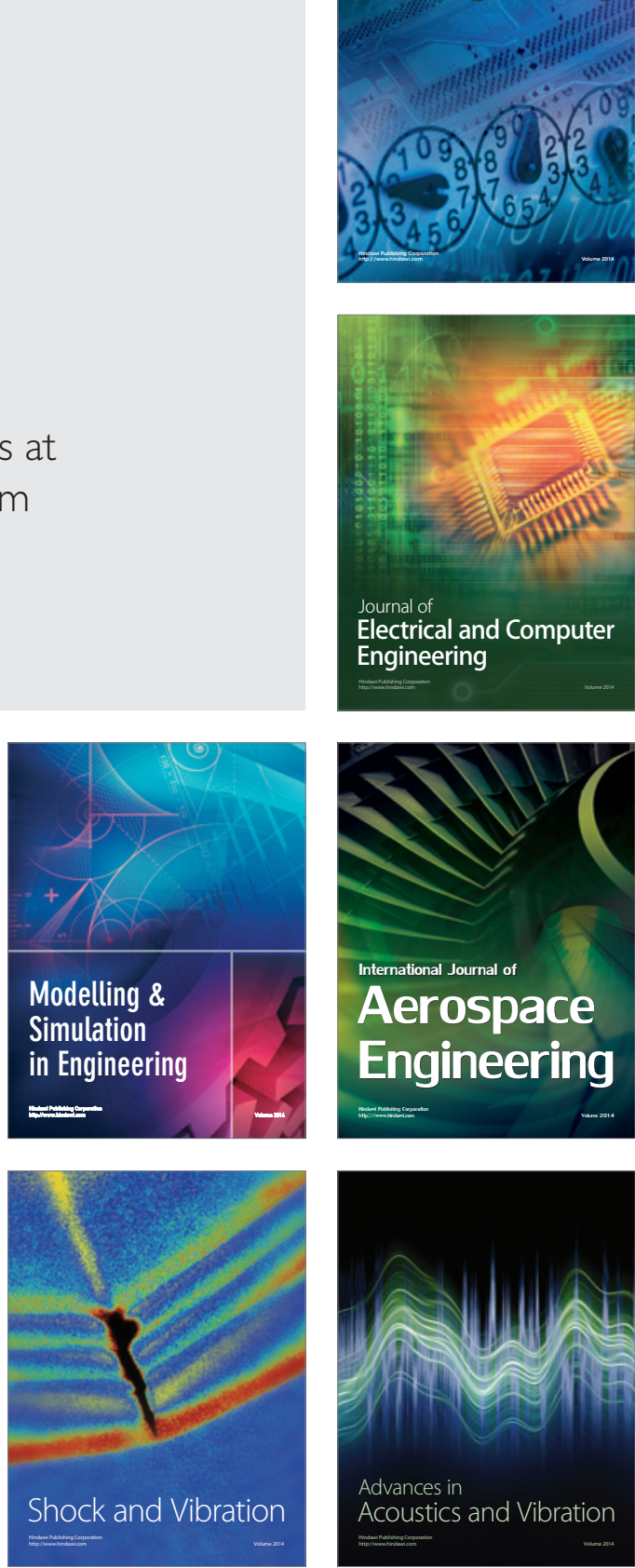